This is the authors' version of the paper.

The final publication has appeared in Monatsh. Math. 164 (2011), 157-170.

\title{
Formulas for the number of gridlines
}

\author{
Anne-Maria Ernvall-Hytönen* Kaisa Matomäki \\ Pentti Haukkanen Jorma K. Merikoski
}

\begin{abstract}
Let $l(n)$ be the number of lines through at least two points of an $n \times n$ rectangular grid. We prove recursive and asymptotic formulas for it using respectively combinatorial and number theoretic methods. We also study the ratio $l(n) / l(n-1)$. All this originates from Mustonen's experimental results.

Keywords: Rectangular grid, Lattice points, Euler $\phi$-function, Asymptotic formulas, Recursive formulas

MSC2010: 05A99, 11B37, 11N37, 11P21
\end{abstract}

\section{Introduction}

Let $l(n)$ be the number of lines through at least two points of an $n \times n$ rectangular grid. Sloane's database ([9], Sequence A018808) mentions an explicit formula

$$
l(n)=\frac{1}{2}\left(f_{1}(n)-f_{2}(n)\right),
$$

where

$$
f_{k}(n)=\sum_{\substack{n<i, j<n \\(i, j)=k}}(n-|i|)(n-|j|)
$$

and $(i, j)$ denotes the greatest common divisor of $i$ and $j$. There is no proof reference in the database but a proof of a generalization to $m \times n$ grids can be found in Mustonen's paper ([5], Section 3).

One would like to have a closed form expression for $l(n)$ instead of (1) which involves a double sum and does not in itself tell us much about the behaviour of $l(n)$. Besides, applying the formula (1) is computationally tedious. This motivated Mustonen [5] to investigate $l(n)$ experimentally and to state various conjectures concerning its behaviour. The aim of this paper is to widen the knowledge about $l(n)$ by proving Mustonen's conjectures.

We will first, in Section 2, prove recursive formulas for $l(n)$ using combinatorial arguments. In particular we show that the recursive formulas which Mustonen predicted in $[5$, Section 6] indeed hold.

*The first author is funded by the Swedish research council Vetenskapsrådet (grant 2009-721) 
Next, in Section 3, we will study $l(n)$ asymptotically. Sheng [8] has shown that $l(n)$ is asymptotically equal to $9 n^{4} /\left(4 \pi^{2}\right)$. We will improve the error term in his asymptotic formula. We will also show that assuming the Riemann hypothesis we obtain a still better error term which corresponds to Mustonen's experimental result ([5], Section 4). Our improvements ultimately depend on known estimates on averages of averages of Euler $\phi$-function.

Finally, in Section 4 , we will study the asymptotic behaviour of the ratio $l(n) / l(n-1)$. We will confirm Mustonen's ([5], Section 4) prediction that the ratio is asymptotically decreasing unless all the prime factors of $n-1$ are large. The proof of this fact utilizes both recursive and asymptotic formulas for $l(n)$.

Before going further, we introduce some notation. Since we consider only integers, we write $[a, b]=\{x \in \mathbb{Z} \mid a \leq x \leq b\}$. Given integers $m, n \geq 2$, we say that a line $l$ is a gridline of the rectangular grid $G=G(m, n)=[0, m-1] \times[0, n-1]$ if it goes through at least two points of $G$. We also say that $l$ then lives in $G$. We write $l(m, n)$ for the number of these gridlines. In particular $l(n, n)=l(n)$.

\section{Recursive formulas for $l(n)$}

We first sketch, how Mustonen ([5], Section 6) experimentally found recursive formulas for $l(n)$ and $l(n-1, n)$.

Since $G(n)=G(n, n)$ can be constructed from $G(n-1)$ by adding first a new column of $n-1$ points and then a new row of $n$ points, it is natural to look for a relation between $l(n), l(n-1, n)$ and $l(n-1)$. Consider the data where $n=3,4, \ldots, 35$. A linear regression analysis suggests that

$$
l(n) \approx 2 l(n-1, n)-l(n-1) .
$$

The residuals

$$
r(n)=l(n)-2 l(n-1, n)+l(n-1)
$$

are strictly increasing except for every fourth $n$ where the same value appears twice. This motivates to study differences

$$
r(n)-r(n-1) .
$$

Indeed Mustonen found a simple representation for this difference (which is (6) below).

To make (4) practically applicable, a recursive formula must be found also for $l(n-$ $1, n)$. Mustonen studied this as well and found analogously to (3) that

$$
l(n-1, n) \approx 2 l(n-1, n-1)-l(n-2, n-1) .
$$

He also found a formula for

$$
s(n)=l(n-1, n)-2 l(n-1, n-1)+l(n-2, n-1)
$$

(see (8) below).

We will rigorously prove the following theorem which shows that Mustonen's experimental formulas indeed hold for all $n \geq 2$. 
Theorem 1. For all $n \geq 2$,

$$
\begin{aligned}
l(n) & =2 l(n-1, n)-l(n-1)+r(n), \\
l(n-1, n) & =2 l(n-1)-l(n-2, n-1)+s(n) .
\end{aligned}
$$

Here

$$
\begin{gathered}
r(n)=r(n-1)+4(\phi(n-1)-e(n)), \\
e(n)= \begin{cases}0 & \text { if } n \text { is even, } \\
\phi\left(\frac{n-1}{2}\right) & \text { if } n \text { is odd, }\end{cases}
\end{gathered}
$$

or explicitly

$$
r(n)=4 \sum_{i=1}^{n-1} \phi(i)-4 \sum_{i=1}^{\left\lfloor\frac{n-1}{2}\right\rfloor} \phi(i)=4 \sum_{i=\left\lfloor\frac{n+1}{2}\right\rfloor}^{n-1} \phi(i),
$$

and

$$
s(n)= \begin{cases}(n-1) \phi(n-1) & \text { if } n \text { is even, } \\ \frac{1}{2}(n-1) \phi(n-1) & \text { if } n \equiv 1(\bmod 4), \\ 0 & \text { if } n \equiv 3(\bmod 4),\end{cases}
$$

and $l(0)=l(0,1)=r(1)=0$ and $l(1)=1$.

Proof. First, we prove (4). Let us call

3-lines of $G(n)$ the lines through exactly three points of $G(n)=[0, n-1] \times[0, n-1]$, one of them in $[1, n-1] \times\{0\}$ and one in $\{0\} \times[1, n-1]$,

2-lines of $G(n)$ the lines through exactly two points of $G(n)$, located in the boundaries of $G(n)$ as above, and

1-lines of $G(n)$ the lines through the origin and exactly one other point of $G(n)$.

To find $l(n)$ recursively, we first add the numbers of lines living in $[1, n-1] \times[0, n-1]$ and, respectively, in $[0, n-1] \times[1, n-1]$. The result is $2 l(n-1, n)$, but certain lines have been counted twice, namely

the $l(n-1)$ lines living in $[1, n-1] \times[1, n-1]$

and

the 3-lines in $G(n)$; let their number be $r_{3}(n)$.

On the other hand, the 2- and 1-lines of $G(n)$ have been ignored; let their numbers be respectively $r_{2}(n)$ and $r_{1}(n)$. In conclusion, we have

$$
l(n)=2 l(n-1, n)-l(n-1)-r_{3}(n)+r_{2}(n)+r_{1}(n) .
$$

We still have to find recursive formulas for $r_{1}, r_{2}$ and $r_{3}$.

Let us study $r_{3}(n)$. All 3-lines in $G(n-1)$ are 3-lines also in $G(n)$. So we obtain $r_{3}(n)$ by adding to $r_{3}(n-1)$ the number of those 3 -lines in $G(n)$ that go through $P=(0, n-1)$ or $Q=(n-1,0)$. 
If $n$ is even, consider a line $l=P S$ or $l=Q T$ where $S \in[0, n-1] \times\{0\}, T \in$ $\{0\} \times[0, n-1]$. Then $l$ meets $G(n)$ at even number of points, and so it is not a 3 -line. Hence $r_{3}(n)=0$.

Now assume that $n$ is odd. A line $l=P S$ is a 3 -line if and only if it meets $G(n)$ at a point $M_{l}=\left(m_{l}, \frac{n-1}{2}\right)$ where $m_{l} \in\left\{1, \ldots, \frac{n-1}{2}\right\}$ satisfies $\operatorname{gcd}\left(m_{l}, \frac{n-1}{2}\right)=1$. The number of such lines is the number of the $m_{l}$ 's, that is $\phi\left(\frac{n-1}{2}\right)$. Since there are also $\phi\left(\frac{n-1}{2}\right)$ 3 -lines $Q T$, we have $r_{3}(n)=2 \phi\left(\frac{n-1}{2}\right)$.

In all,

$$
r_{3}(n)=r_{3}(n-1)+ \begin{cases}0 & \text { if } n \text { is even, } \\ 2 \phi\left(\frac{n-1}{2}\right) & \text { if } n \text { is odd. }\end{cases}
$$

Using similar ideas, it can be shown that

$$
r_{2}(n)=r_{2}(n-1)+2 \phi(n-1)
$$

and

$$
r_{1}(n)=r_{1}(n-1)+2 \phi(n-1)- \begin{cases}0 & \text { if } n \text { is even, } \\ 2 \phi\left(\frac{n-1}{2}\right) & \text { if } n \text { is odd. }\end{cases}
$$

Substituting (10), (11), and (12) in (9), we obtain (4).

Second, we prove (5). Consider $G(n, n-1)=[0, n-1] \times[0, n-2]$. To find $l(n-1, n)$ recursively, we first add the numbers of lines living in $G(n-1)$ and, respectively, in $[1, n-$ $1] \times[0, n-2]$. The result is $2 l(n-1)$, but the following lines have been counted twice:

the $l(n-2, n-1)$ lines living in $[1, n-2] \times[0, n-2]$

and

the lines going through a point $P \in\{0\} \times[0, n-2]$, a point $Q \in\{n-1\} \times[0, n-2]$ and exactly one other point of $G(n, n-1)$; let their number be $s_{2}(n)$.

On the other hand, such lines $P Q$ that do not meet $G(n, n-1)$ in any other point have been ignored; let their number be $s_{1}(n)$. Now

$$
l(n-1, n)=2 l(n-1)-l(n-2, n-1)-s_{2}(n)+s_{1}(n) .
$$

Let us study $s_{1}(n)$. Choose first $P=(0, i)$. The point $Q=(n-1, j)$ applies if and only if $(i-j, n-1)=1$. Since the number of such $Q$ 's is $\phi(n-1)$, there are $\phi(n-1)$ acceptable lines through $P$. Because $P$ can be chosen in $n-1$ ways, we have

$$
s_{1}(n)=(n-1) \phi(n-1) .
$$

A similar reasoning gives

$$
s_{2}(n)= \begin{cases}0 & \text { if } n \text { is even } \\ (n-1) \phi\left(\frac{n-1}{2}\right) & \text { if } n \text { is odd }\end{cases}
$$

Now (5) follows by substituting (15) and (14) in (13).

Finally, we note that $(7)$ can be shown simply by solving $r(n)$ from the recursive equation (6) with initial condition $r(1)=0$. 
We now unite (4) and (5) into a single recursive formula for $l(n)$ only.

Theorem 2. For all $n \geq 2$,

$$
l(n)=l(n-1)+2 \sum_{i=1}^{n} s(i)+2 \sum_{i=1}^{n-1} r(i)+r(n) .
$$

Proof. By adding (4) and (5), we obtain

$$
l(n)=l(n-1)+l(n-1, n)-l(n-2, n-1)+r(n)+s(n),
$$

and further, replacing $n$ with $n-1, n-2, \ldots, 2$ gives

$$
l(n)=l(n-1, n)+\sum_{i=1}^{n}(r(i)+s(i)) .
$$

On the other hand, by (4),

$$
l(n-1)=2 l(n-2, n-1)-l(n-2)+r(n-1) .
$$

Adding this to (5) and proceeding as above yields

$$
l(n-1, n)=l(n-1)+l(n-2, n-1)-l(n-2)+r(n-1)+s(n),
$$

and further

$$
l(n-1, n)=l(n-1)+\sum_{i=1}^{n-1} r(i)+\sum_{i=1}^{n} s(i) .
$$

Substituting (18) into (17) gives (16).

\section{An asymptotic formula for $l(n)$}

Asymptotic behaviour of $l(n)$ has been studied by Sheng [8]. His more general Lemma 7 implies the following result.

Theorem 3. For all $n \geq 2$,

$$
l(n)=\frac{3 n^{4}}{8 \zeta(2)}+O\left(n^{3} \log n\right)=\frac{9 n^{4}}{4 \pi^{2}}+O\left(n^{3} \log n\right) .
$$

Here $\zeta(s)$ denotes Riemann's zeta function.

In this paper we are able to improve the error term as follows.

Theorem 4. Let $n \geq 2$. Then

$$
l(n)=\frac{9 n^{4}}{4 \pi^{2}}+O\left(n^{3} \exp \left(-A(\log n)^{\frac{3}{5}}(\log \log n)^{-\frac{1}{5}}\right)\right)
$$

for certain constant $A>0$. Assuming the Riemann hypothesis we have

$$
l(n)=\frac{9 n^{4}}{4 \pi^{2}}+O\left(n^{\frac{5}{2}+\varepsilon}\right)
$$

for any $\varepsilon>0$. 
Based on his experiments, Mustonen ([5], Section 4) has predicted earlier that (20) indeed holds.

We prove Theorem 4 using the presentation (1) for $l(n)$, so we study $f_{k}(n)$ defined by (2). We need the following elementary lemma (which is [1, Exercise 2.16]).

Lemma 5. For all $n \geq 2$,

$$
\sum_{\substack{i=1 \\(i, n)=1}}^{n} i=\frac{1}{2} n \phi(n)
$$

Proof. Simply note that

$$
2 \sum_{\substack{i=1 \\(i, n)=1}}^{n} i=\sum_{\substack{i=1 \\(i, n)=1}}^{n} i+\sum_{\substack{i=1 \\(i, n)=1}}^{n}(n-i)=\sum_{\substack{i=1 \\(i, n)=1}}^{n} n=n \phi(n) .
$$

Now we are able to dispose of the double summation in (2).

Lemma 6. Let $k \geq 1$. For all $n \geq 2$,

$$
f_{k}(n+1)=8 \sum_{i=1}^{\lfloor n / k\rfloor}(n+1-k i)\left(n+1-\frac{k i}{2}\right) \phi(i)
$$

Proof. We have

$$
\begin{aligned}
f_{k}(n+1)= & \sum_{\substack{-n \leq i, j \leq n \\
(i, j)=k}}(n+1-k|i|)(n+1-k|j|) \\
= & 4 \sum_{\substack{1 \leq i, j \leq\lfloor n / k\rfloor \\
(i, j)=1}}^{\substack{\lfloor n / k\rfloor\\
}}(n+1-k i)(n+1-k j)+4(n+1-k)(n+1) \\
& \quad \sum_{i=2}^{i}(n+1-k i) \sum_{\substack{j=1 \\
(j, i)=1}}^{i}(n+1-k j) \\
& +4(n+1-k)^{2}+4(n+1-k)(n+1) .
\end{aligned}
$$

By Lemma 5 we see that

$$
\begin{aligned}
f_{k}(n+1) & =8 \sum_{i=2}^{\lfloor n / k\rfloor}(n+1-k i)\left(n+1-\frac{k i}{2}\right) \phi(i)+4(n+1-k)(2 n+2-k) \\
& =8 \sum_{i=1}^{\lfloor n / k\rfloor}(n+1-k i)\left(n+1-\frac{k i}{2}\right) \phi(i) .
\end{aligned}
$$


Next we apply the partial summation formula

$$
\sum_{i=1}^{N} a_{i} b_{i}=\left(\sum_{i=1}^{N} a_{i}\right) b_{N}-\sum_{i=1}^{N-1}\left(\sum_{j=1}^{i} a_{j}\right)\left(b_{i+1}-b_{i}\right)
$$

to reach sums

$$
\Phi(k)=\sum_{i=1}^{k} \phi(i)
$$

Lemma 7. For all $n \geq 2$,

$$
f_{1}(n+1)=4(n+2) \sum_{i=1}^{n} \Phi(i)+8 \sum_{i=1}^{n-1} \sum_{j=1}^{i} \Phi(j) .
$$

If $n$ is odd, then

$$
f_{2}(n+1)=8(n+3) \sum_{i=1}^{\frac{n-1}{2}} \Phi(i)+32 \sum_{i=1}^{\frac{n-3}{2}} \sum_{j=1}^{i} \Phi(j) .
$$

If $n$ is even, then

$$
f_{2}(n+1)=4(n+2) \Phi\left(\frac{1}{2} n\right)+8(n+5) \sum_{i=1}^{\frac{n}{2}-1} \Phi(i)+32 \sum_{i=1}^{\frac{n}{2}-2} \sum_{j=1}^{i} \Phi(j) .
$$

Proof. By Lemma 6

$$
f_{1}(n+1)=8 \sum_{i=1}^{n+1}(n+1-i)\left(n+1-\frac{1}{2} i\right) \phi(i) .
$$

To show (22), we start by applying partial summation (21) with

$$
N=n+1, \quad a_{i}=\phi(i), \quad b_{i}=(n+1-i)\left(n+1-\frac{1}{2} i\right) .
$$

This gives

$$
\begin{aligned}
f_{1}(n+1) & =-8 \sum_{i=1}^{n}\left\{(n-i)\left[n+1-\frac{1}{2}(i+1)\right]-(n+1-i)\left(n+1-\frac{1}{2} i\right)\right\} \Phi(i) \\
& =8 \sum_{i=1}^{n}\left(\frac{3}{2} n-i+1\right) \Phi(i) .
\end{aligned}
$$

Then we apply partial summation (21) with

$$
N=n, \quad a_{i}=\Phi(i), \quad \text { and } \quad b_{i}=\frac{3}{2} n-i+1
$$


getting

$$
\begin{aligned}
f_{1}(n+1) & =8\left(\frac{1}{2} n+1\right) \sum_{i=1}^{n} \Phi(i)-8 \sum_{i=1}^{n-1}(-1) \sum_{j=1}^{i} \Phi(j) \\
& =4(n+2) \sum_{i=1}^{n} \Phi(i)+8 \sum_{i=1}^{n-1} \sum_{j=1}^{i} \Phi(j) .
\end{aligned}
$$

Similarly, for even $n$, we get

$$
\begin{aligned}
f_{2}(n+1) & =8 \sum_{i=1}^{\frac{n}{2}}(n+1-2 i)(n+1-i) \phi(i) \\
& =8\left(\frac{n}{2}+1\right) \Phi\left(\frac{n}{2}\right)+8 \sum_{i=1}^{\frac{n}{2}-1}(3 n-4 i+1) \Phi(i) \\
& =4(n+2) \Phi\left(\frac{n}{2}\right)+8(n+5) \sum_{i=1}^{\frac{n}{2}-1} \Phi(i)+8 \cdot 4 \sum_{i=1}^{\frac{n}{2}-2} \sum_{j=1}^{i} \Phi(j) .
\end{aligned}
$$

The case of odd $n$ can be handled analogously.

Remark 8. Lemma 7 would also follow from Theorem 2. However we have decided to take a more analytic path in proving the asymptotic formula.

Let us now define

$$
E_{\Phi}(n)=\Phi(n)-\frac{3 n^{2}}{\pi^{2}} .
$$

At this point we would get Sheng's result (Theorem 3) by applying the following classical result (see e.g. [4], § I.21).

Lemma 9. For all $n \geq 2$,

$$
E_{\Phi}(n)=O(n \log n) .
$$

This has been improved and so we could already reach a refinement of Theorem 3 .

Lemma 10 (Walfisz [12], p. 144, Satz 1). For all $n \geq 2$,

$$
E_{\Phi}(n)=O\left(n(\log n)^{\frac{2}{3}}(\log \log n)^{\frac{4}{3}}\right) .
$$

Saltykov [7] presented a sharper formula but its correctness was controversial; see [2], p. 314. Finally Pétermann ([6], Section 5) falsified it.

To continue our journey toward proof of Theorem 4, we consider averages of averages of Euler $\phi$-function. To this end we define

$$
E_{R}(n)=\sum_{i=1}^{n} E_{\Phi}(i)-\frac{3 n^{2}}{2 \pi^{2}} .
$$

Next we express $f_{1}$ and $f_{2}$ using $E_{\Phi}$ and $E_{R}$. 
Lemma 11. For all $n \geq 2$,

$$
f_{1}(n+1)=\frac{6}{\pi^{2}}(n+1)^{4}+4(n+2) E_{R}(n)+8 \sum_{i=1}^{n-1} E_{R}(i)+O\left(n^{2}\right) .
$$

If $n$ is odd, then

$$
f_{2}(n+1)=\frac{3}{2 \pi^{2}}(n+1)^{4}+8(n+3) E_{R}\left(\frac{n-1}{2}\right)+32 \sum_{i=1}^{\frac{n-3}{2}} E_{R}(i)+O\left(n^{2}\right) .
$$

If $n$ is even, then

$$
\begin{aligned}
f_{2}(n+1)= & \frac{3}{2 \pi^{2}}(n+1)^{4}+8(n+5) E_{R}\left(\frac{1}{2} n-1\right) \\
& +32 \sum_{i=1}^{\frac{n}{2}-2} E_{R}(i)+4(n+2) E_{\Phi}\left(\frac{1}{2} n\right)+O\left(n^{2}\right) .
\end{aligned}
$$

Proof. By definitions of $E_{\Phi}(n)$ and $E_{R}(n)$ we have

$$
\begin{aligned}
\sum_{k=1}^{K} \Phi(k) & =\sum_{k=1}^{K}\left(\frac{3 k^{2}}{\pi^{2}}+E_{\Phi}(k)\right)=\frac{3}{\pi^{2}} \cdot \frac{K(K+1)(2 K+1)}{6}+\frac{3 K^{2}}{2 \pi^{2}}+E_{R}(K) \\
& =\frac{K^{3}+3 K^{2}}{\pi^{2}}+E_{R}(K)+O(K)
\end{aligned}
$$

and

$$
\begin{aligned}
\sum_{l=1}^{L} \sum_{k=1}^{l} \Phi(k) & =\sum_{l=1}^{L}\left(\frac{l^{3}+3 l^{2}}{\pi^{2}}+E_{R}(l)+O(l)\right) \\
& =\frac{L^{2}(L+1)^{2}}{4 \pi^{2}}+\frac{L(L+1)(2 L+1)}{2 \pi^{2}}+\sum_{l=1}^{L} E_{R}(l)+O\left(L^{2}\right) \\
& =\frac{L^{4}+6 L^{3}}{4 \pi^{2}}+\sum_{l=1}^{L} E_{R}(l)+O\left(L^{2}\right) .
\end{aligned}
$$

The claims follow by substituting these into Lemma 7 .

Replacing $n$ with $n-1$, we have the following

Theorem 12. Let $n \geq 3$. Define $E_{l}(n)$ by

$$
l(n)=\frac{9 n^{4}}{4 \pi^{2}}+E_{l}(n)
$$

If $n$ is even, then

$$
\begin{aligned}
E_{l}(n)=2(n+1) E_{R}(n-1)-4(n+2) E_{R}\left(\frac{1}{2} n-1\right) \\
-12 \sum_{i=1}^{\frac{n}{2}-2} E_{R}(i)+4 \sum_{i=\frac{n}{2}-1}^{n-2} E_{R}(i)+O\left(n^{2}\right) .
\end{aligned}
$$


If $n$ is odd, then

$$
\begin{aligned}
E_{l}(n)=2(n+1) E_{R}(n-1)-4(n+4) E_{R}\left(\frac{n-3}{2}\right) \\
\quad-12 \sum_{i=1}^{\frac{n-5}{2}} E_{R}(i)+4 \sum_{i=\frac{n-3}{2}}^{n-2} E_{R}(i)-2(n+1) E_{\Phi}\left(\frac{n-1}{2}\right)+O\left(n^{2}\right) .
\end{aligned}
$$

In a sense, (26), (27) and (28) together give the best possible asymptotic formula for $l(n)$. But to apply it in practice requires knowledge about $E_{\Phi}$ and $E_{R}$. We already mentioned results concerning $E_{\Phi}$. Next lemma gives us the necessary information about $E_{R}$.

Lemma 13. Let $n \geq 2$. Then

$$
E_{R}(n)=O\left(n^{2} \exp \left[-A(\log n)^{\frac{3}{5}}(\log \log n)^{-\frac{1}{5}}\right]\right)
$$

for certain constant $A>0$. Furthermore,

$$
E_{R}(n)=O\left(n^{\frac{3}{2}+\varepsilon}\right)
$$

for all $\varepsilon>0$ if and only if the Riemann hypothesis is true.

Proof. The first result is due to Suryanarayana and Sitaramachandra Rao [11, eq. (1.11)]. The if-part of the second claim was proved by Suryanarayana [10, eq. (3.41)] (there should be $2 \pi^{2}$ instead of $\pi^{2}$ there). The converse was shown to hold by Codecà [3].

Now we can turn to $E_{l}$. By Theorem 12,

$$
E_{l}(n)=O\left(\max _{m \leq n}\left(n\left|E_{R}(m)\right|+n\left|E_{\Phi}(m)\right|\right)+n^{2}\right) .
$$

Theorem 4 follows now from Lemmas 9 and 13.

Conversely, does (20) imply the Riemann hypothesis? This would give an interesting geometric characterization of the Riemann hypothesis. Techniques used in [3] are likely to work also in this case.

\section{The ratio $l(n) / l(n-1)$}

Mustonen ([5], Section 4) showed experimentally that the function $\frac{l(n)}{l(n-1)}$ is asymptotically decreasing unless all the prime factors of $n-1$ are large. We prove this conjecture:

Theorem 14. If

$$
\frac{l(n)}{l(n-1)}>\frac{l(n-1)}{l(n-2)}
$$

then $n$ is even and

$$
\frac{\phi(n-1)}{n}>\frac{9}{\pi^{2}}+o(1)
$$


Let $p_{1}, \ldots, p_{k}$ be the distinct prime factors of $n-1$. Then,

$$
\frac{\phi(n-1)}{n} \approx \frac{\phi(n-1)}{n-1}=\left(1-\frac{1}{p_{1}}\right) \cdots\left(1-\frac{1}{p_{k}}\right),
$$

and so (30) is asymptotically equivalent with

$$
\left(1-\frac{1}{p_{1}}\right) \cdots\left(1-\frac{1}{p_{k}}\right)>\frac{9}{\pi^{2}} .
$$

of Theorem 14. Consider the difference

$$
\frac{l(n)}{l(n-1)}-\frac{l(n-1)}{l(n-2)}=\frac{l(n) l(n-2)-l(n-1)^{2}}{l(n-2) l(n-1)} .
$$

As the denominator is positive, it is enough to study the numerator. By Theorem 2,

$$
l(n)-l(n-1)=l(n-1)-l(n-2)+2 s(n)+r(n-1)+r(n) .
$$

Hence

$$
\begin{aligned}
l(n) l(n-2)-l(n-1)^{2} & =l(n)(l(n-2)-l(n-1))+l(n-1)(l(n)-l(n-1)) \\
& =l(n)(2 s(n)+r(n-1)+r(n))-(l(n)-l(n-1))^{2} .
\end{aligned}
$$

To continue, notice that (7) and (25) imply $r(n)=\frac{9 n^{2}}{\pi^{2}}+O\left(E_{\Phi}(n)\right)$, and so

$$
r(n-1)+r(n)=\frac{18 n^{2}}{\pi^{2}}+O\left(\left|E_{\Phi}(n)\right|+\left|E_{\Phi}(n-1)\right|\right) .
$$

Also, we have

$$
l(n)-l(n-1)=\frac{9 n^{3}}{\pi^{2}}+O\left(\left|E_{l}(n)\right|+\left|E_{l}(n-1)\right|\right)
$$

Consequently,

$$
\begin{aligned}
l(n)(2 s(n) & +r(n-1)+r(n))-(l(n)-l(n-1))^{2}=-\frac{81}{2 \pi^{4}} n^{6}+\frac{9}{2 \pi^{2}} n^{4} s(n) \\
& +O\left(n^{4}\left(\left|E_{\Phi}(n)\right|+\left|E_{\Phi}(n-1)\right|\right)\right)+O\left(n^{3}\left(\left|E_{l}(n)\right|+\left|E_{l}(n-1)\right|\right)\right) \\
& +O\left(\left(\left|E_{\Phi}(n)\right|+\left|E_{\Phi}(n-1)\right|\right) E_{l}(n)\right)+O\left(E_{l}(n)^{2}+E_{l}(n-1)^{2}\right) .
\end{aligned}
$$

We know from Section 3 that

$$
E_{\Phi}(n) n^{4}, E_{l}(n) n^{3}, E_{\Phi}(n) E_{l}(n), E_{l}(n)^{2}=o\left(n^{6}\right),
$$

so (29) can hold only if the main term is $>-o\left(n^{6}\right)$. If $n$ is even, substituting $s(n)=$ $(n-1) \phi(n-1)=n \phi(n-1)+O(n)$ we get the asymptotic condition:

$$
\frac{\phi(n-1)}{n}>\frac{9}{\pi^{2}}
$$

On the other hand, if $n$ is odd, then $s(n)=\frac{1}{2}(n-1) \phi(n-1)$ or $=0$ depending on the residue of $n$ modulo 4. Asymptotically, the condition never holds as

$$
\frac{9}{\pi^{2}}>\frac{\phi(n-1)}{2 n} \text {. }
$$

Experiments also falsify (29) for small odd values of $n$. 


\section{References}

[1] T. M. Apostol, Introduction to Analytic Number Theory. Springer, 1976. Third printing, 1986.

[2] E. A. Bender, O. Patashnik and H. Rumsey Jr., Pizza slicing, phi's and the Riemann hypothesis. Amer. Math. Monthly 101 (1994), 307-317.

[3] P. Codecà, A note on Euler's $\phi$-function. Ark. Mat. 19 (1981), 261-263.

[4] D. S. Mitrinović, J. Sándor and B. Crstici, Handbook of Number Theory. Kluwer, 1996.

[5] S. Mustonen, On lines and their intersection points in a rectangular grid of points. http://www.survo.fi/papers/PointsInGrid.pdf

[6] Y.-F. S. Pétermann, On an estimate of Walfisz and Saltykov for an error term related to the Euler function. J. Théor. Nombr. Bordeaux 10 (1998), 203-236.

[7] A. I. Saltykov, On Euler's function. (In Russian.) Vestnik Moskov. Univ. Ser. I Mat. Meh. 1960 no. 6, 34-50.

[8] T. K. Sheng, Lines determined by lattice points in $\mathrm{R}^{2}$. Nanta Math. 10 (1977), 77-81.

[9] N. J. A. Sloane, The On-Line Encyclopedia of Integer Sequences.

http://www.research.att.com/ njas/sequences/

[10] D. Suryanarayana, On the average order of the function $E(x)=\sum_{n \leq x} \phi(n)-$ $3 x^{2} / \pi^{2}$. II. J. Indian Math. Soc. (N.S.) 42 (1978), 195-197.

[11] D. Suryanarayana and R. Sitaramachandra Rao, On the average order of the function $E(x)=\sum_{n \leq x} \phi(n)-3 x^{2} / \pi^{2}$. Ark. Mat. 10 (1972), 99-106.

[12] A. Walfisz, Weylsche Exponentialsummen in der neueren Zahlentheorie. VEB Deutsch. Verl. Wiss., 1963.

A-M. Ernvall-Hytönen

Institutionen för matematik

Kungliga Tekniska Högskolan

10044 Stockholm, Sweden.

hytonen@kth.se

K. Matomäki

Department of Mathematics

20014 University of Turku

Finland.

ksmato@utu.fi

P. Haukkanen and J. K. Merikoski 
Department of Mathematics and Statistics 33014 University of Tampere

Finland

pentti.haukkanen@uta.fi and jorma.merikoski@uta.fi 